\title{
Family physicians' perception on environmental influences in promoting physical activity in their obese patients
}

\author{
Samuel N. Forjuoh ${ }^{1,2^{\star}}$, Chanam Lee ${ }^{3}$, Suojin Wang ${ }^{4}$, Yan Hong ${ }^{2}$, Marcia G. Ory ${ }^{2}$ \\ ${ }^{1}$ Department of Family \& Community Medicine, Scott \& White Healthcare, College of Medicine, Texas A\&M Health Science Cen- \\ ter, Temple, USA; ${ }^{*}$ Corresponding Author: sforjuoh@sw.org \\ ${ }^{2}$ Department of Health Promotion \& Community Health Sciences, School of Rural Public Health, Texas A\&M Health Science Center, \\ College Station, USA \\ ${ }^{3}$ Department of Landscape Architecture \& Urban Planning, College of Architecture, Texas A\&M University, College Station, USA \\ ${ }^{4}$ Department of Statistics, College of Science, Texas A\&M University, College Station, USA
}

Received 13 August 2012; revised 21 September 2012; accepted 28 October 2012

\section{ABSTRACT}

Although many obese patients do not engage in adequate physical activity (PA), little is known about whether physicians discuss environmental barriers in promoting PA. We conducted this study to describe physicians' views about discussing environmental barriers in promoting PA with obese patients and determine whether counseling varies by years of practice. We conducted an on-line survey of family physicians practicing in 17 clinics in Central Texas. We performed descriptive analysis on barriers to PA, counseling on meeting PA recommendation, and written prescription for exercise and bivariate analysis by years of practice. Statistical significance was set at $p<0.05$. Of 96 physicians invited, 57 (59.4\%) completed the survey. Majority reported their obese patients brought up the environment as a barrier to walking (89.3\%) or PA (91.2\%). Majority $(80.7 \%)$ also reported asking about environmental barriers to walking, with $84.2 \%$ giving specific examples to overcome these barriers. While $96.5 \%$ reported advising their obese patients about meeting the PA recommendation, only $26.3 \%$ reported giving them a written prescription for exercise. These did not vary significantly by years of practice. Physicians felt having more time with their patients and knowing specific strategies to overcome environmental barriers, safe places in patients' neighborhoods, and what environmental factors influence PA may help discuss environmental issues with their obese patients. Physicians want and counsel their obese patients to be physically active, but do not generally give prescriptions for exer- cise irrespective of years of practice. They also want tools to enhance their abilities to better assist these patients.

Keywords: Barriers to Physical Activity; Environmental Barriers; Obesity; Physical Activity; Physician Counseling; Physician Practices; Walking

\section{INTRODUCTION}

Obesity is a growing health problem worldwide. It affects Americans of all ages across the entire nation [1]. Current estimates indicate that almost a third of adult Americans are obese [2], with rates disproportionately high among certain minority and ethnic groups and those characterized by lower socioeconomic status [3]. Although moderate exercise such as walking can help prevent or manage health problems associated with obesity [4], many obese individuals do not engage in adequate physical activity [5]. Currently, less than half of American adults are estimated to obtain the recommended physical activity level of a minimum of 30 minutes of physical activity daily for at least five days per week [3]. A 2003 US Physical Activity Study found that nationally only $34 \%$ of the population were regular walkers who met the recommended physical activity level, 46\% were occasional walkers, and $21 \%$ were not walking at all [6]. These statistics mirror data on physician counseling of their patients about exercise, which is low nationally at an estimated $30 \%$ - 40\% depending on assessment methodologies and populations being examined [7-9].

The physical environment may have a major influence on physical activity by acting both as a barrier and as a facilitator [10]. In particular, forms (e.g., sidewalks and street network patterns), uses (e.g., shops and recreational facilities) and the characteristics (e.g., safety and 
lighting) of the built environment can influence physical activity, especially walking [11,12]. Getting injured due to an environmental hazard may limit one's desire to engage in physical activities, for example, fracturing a limb from a fall. Similarly getting mugged due to lack of adequate lighting in one's environment may severely limit one's desire to go out for a walk. While environmental interventions may not necessarily enhance physical activity for everyone, they may be particularly effective for certain segments of the population such as those who are less healthy, unemployed, or retired [13]. Further, environmental inequities have been linked to health disparities related to obesity and diabetes [14].

Healthcare providers have a role in promoting physical activity. Physicians generally recommend increasing physical activity to patients, albeit less so to their overweight and obese patients [15]. Among the reasons cited by physicians for counseling only a minority of patients include time constraints, lack of resources to effect behavioral changes, lack of reimbursement, limited training in appropriate behavioral change counseling, and perceived lack of interest from patients [9]. In hopes of contributing to the development of best practice tools for use by physicians to counsel their patients to help overcome environmental barriers to physical activity, we designed this study to examine the extent to which primary care physicians encourage walking and other physical activity to their patients, and are aware of and address environmental facilitators or barriers.

\section{METHODS}

\subsection{Study Design and Setting}

Working in collaboration with the Central Texas Primary Care Research Network (CenTexNet), a primary care Practice-Based Research Network (PBRN) based in Temple, Texas, we conducted a cross-sectional on-line survey using the website of the Agency for Healthcare Research and Quality's PBRN Resource Center. The study setting was 17 primary care clinics of a large university-affiliated, multi-specialty group practice associated with a 250,000-member health maintenance organization in Central Texas. All the clinics were affiliated with CenTexNet and were staffed by a total of 100 family physicians. The study was conducted from October of 2008 through January of 2009. Both the Scott \& White Healthcare and the Texas A\&M University Institutional Review Boards reviewed and approved the study protocol. No written informed consent was sought from subjects for study participation.

\subsection{Study Subjects}

Study subjects were all family physicians practicing in the 17 primary care clinics, who were all members of
CenTexNet. They were enrolled voluntarily and provided a link to the PBRN Resource Center website to access the survey via e-mail.

\subsection{Data Collection Tools}

To inform the development of our survey instrument, we visited with our network physicians during one of their monthly meetings to solicit their ideas and opinions on areas of particular interest to them regarding obesity, physical activity, and the physical environment. We also discussed their awareness of environmental barriers that might affect their recommendations for increasing physical activity, as well as the survey formatting and length. Based on the visit, the survey instrument was designed to obtain data on: 1) physicians' opinions about physical activity and neighborhood environments; 2) physicians' interactions with their typical obese patients regarding physical activity during their last few clinic encounters; 3) their assessments of obese patients' engagement in physical activity and walking specifically; 4) physicians' likelihood of bringing up environmental issues and concerns related to physical activity and walking during clinic encounters with their obese patients; 5) physicians' perceptions about environmental barriers to physical activity and walking for obese patients; 6) physicians' perceptions about good walking environments for obese patients; and 7) physicians' own physical activity behaviors and practices.

The final survey instrument included ordinal-scale questions such as "Strongly agree to strongly disagree" (4 items), "Often-Sometimes-Never" (3 items), and "Very important-Somewhat important-Not at all important" (3 items), "Yes-No" (2 items), and open-ended questions, along with questions on basic demographic data. No individually identifiable information such as name and age was collected. A copy of the contents of the survey can be obtained from the lead author.

\subsection{Statistical Analysis}

Data management and analysis were performed using SPSS version 17. We first compared the respondents and non-respondents using the chi-square statistic. We then performed descriptive analysis on barriers to PA, counseling on meeting PA recommendation, and written prescription for exercise and bivariate analysis by years of practice. Statistical significance was set at $p<0.05$. Given the small sample size and lack of adequate descriptive information about the physicians, we were not able to perform more multivariate analyses.

\section{RESULTS}

\subsection{Survey Response Rates}

Of all the 100 physicians initially invited to participate 
in the study, four were deemed ineligible due to one respondent being on maternity leave, one leaving their practice, one not providing continuity care, and one being a dietician. Of the 96 eligible physicians, 57 completed the survey resulting in a 59.4\% response rate. Respondents were similar to non-respondents by gender, with $70.2 \%$ of respondents being male versus $71.8 \%$ of non-respondents $(p=0.95)$.

\subsection{Physician Characteristics}

Table 1 shows the characteristics of the final study

Table 1. Physician characteristics ( $n=57)$, central Texas survey, 2009.

\begin{tabular}{|c|c|c|}
\hline Characteristic & & $n(\%)$ \\
\hline \multirow{2}{*}{ Gender } & Male & $40(70.2)$ \\
\hline & Female & 17 (29.8) \\
\hline \multirow{7}{*}{ Clinic } & Other & 25 (43.9) \\
\hline & A & $10(17.5)$ \\
\hline & B & $8(14.0)$ \\
\hline & $\mathrm{C}$ & 7 (12.3) \\
\hline & $\mathrm{D}$ & 7 (12.3) \\
\hline & $1-5$ & 10 (17.9) \\
\hline & $6-10$ & $11(19.6)$ \\
\hline \multirow{7}{*}{ Total years of medical practice } & $11-15$ & $6(10.7)$ \\
\hline & $16-20$ & $17(30.4)$ \\
\hline & $21-25$ & $9(16.0)$ \\
\hline & $26-30$ & $3(5.4)$ \\
\hline & 0 & $1(2.1)$ \\
\hline & 1 & $0(0.0)$ \\
\hline & 2 & $3(6.3)$ \\
\hline \multirow{8}{*}{$\begin{array}{c}\text { Physical activity } \\
\text { (days/week active } 30+\text { min) } \\
\text { Mean }=4.7, \mathrm{SD}=1.8\end{array}$} & 3 & $9(18.8)$ \\
\hline & 4 & 8 (16.7) \\
\hline & 5 & $9(18.8)$ \\
\hline & 6 & $7(14.6)$ \\
\hline & 7 & 11 (22.9) \\
\hline & 0 & $4(8.2)$ \\
\hline & 1 & $5(10.2)$ \\
\hline & 2 & $7(14.3)$ \\
\hline \multirow{5}{*}{$\begin{array}{l}\text { Walking (days/week walked) } \\
\text { Mean }=3.8, \mathrm{SD}=2.3\end{array}$} & 3 & $8(16.3)$ \\
\hline & 4 & $4(8.2)$ \\
\hline & 5 & $6(12.2)$ \\
\hline & 6 & $6(12.2)$ \\
\hline & 7 & 9 (18.4) \\
\hline
\end{tabular}

sample. All 57 respondents were family physicians, mainly men. The majority had practiced for more than 15 years. They reported a mean of 4.7 (median $=5$; range $=0$ to 7 ) days of physical activity of at least 30 minutes per week. They also reported a mean of 3.8 (median $=4$; range 0 to 7) days of walking per week.

\subsection{Physician Perception on Physical Activity and the Neighborhood Environment}

Figure 1 summarizes the physicians' perception about physical activity and the neighborhood environment. The vast majority, about 4 out of 5, strongly agreed that walking is a good way of getting physical activity, that physical activity can result in significant health benefits, and that it was their role to discuss physical activity during the medical encounter with their obese patients. There was less strong agreement but still general agreement among physicians that it was their role to address barriers to physical activity in the medical encounter. Similarly, there was a general agreement among physiccians that it was their role to bring up environmental barriers (40.4\% strongly agreed and 31.6\% agreed). Combining strongly agreed and agreed responses indicated that the majority of physicians generally agreed that their obese patients were able to participate in walking activity and that the neighborhood environments and safety concerns could facilitate or deter patients from being physically active.

\subsection{Enabling Factors for Physician Discussion of Environmental Issues with Obese Patients}

Physicians also identified many enabling factors for physician discussion of environmental issues (Figure 2). Time and knowledge were enabling factors. Concerns that the environment cannot be changed or that their obese patients would not be interested in environmental issues related to physical activity and walking were not reported as significant dissuaders to discussion.

\subsection{Physician Interaction with the Typical Obese Patient during an Encounter}

Regarding physicians' clinical practice with their obese patients (table not shown), the vast majority reported affirmatively (yes/no responses) to helping their obese patients to set specific goals to improve exercise (89.5\%), to make a treatment plan $(89.5 \%)$, and to set specific goals to improve their eating habits (86.0\%). In this context, still only a minority of physicians reported providing their obese patients a written prescription for exercise (24.6\%) or a written prescription for specific types of exercise such as walking (26.3\%). 


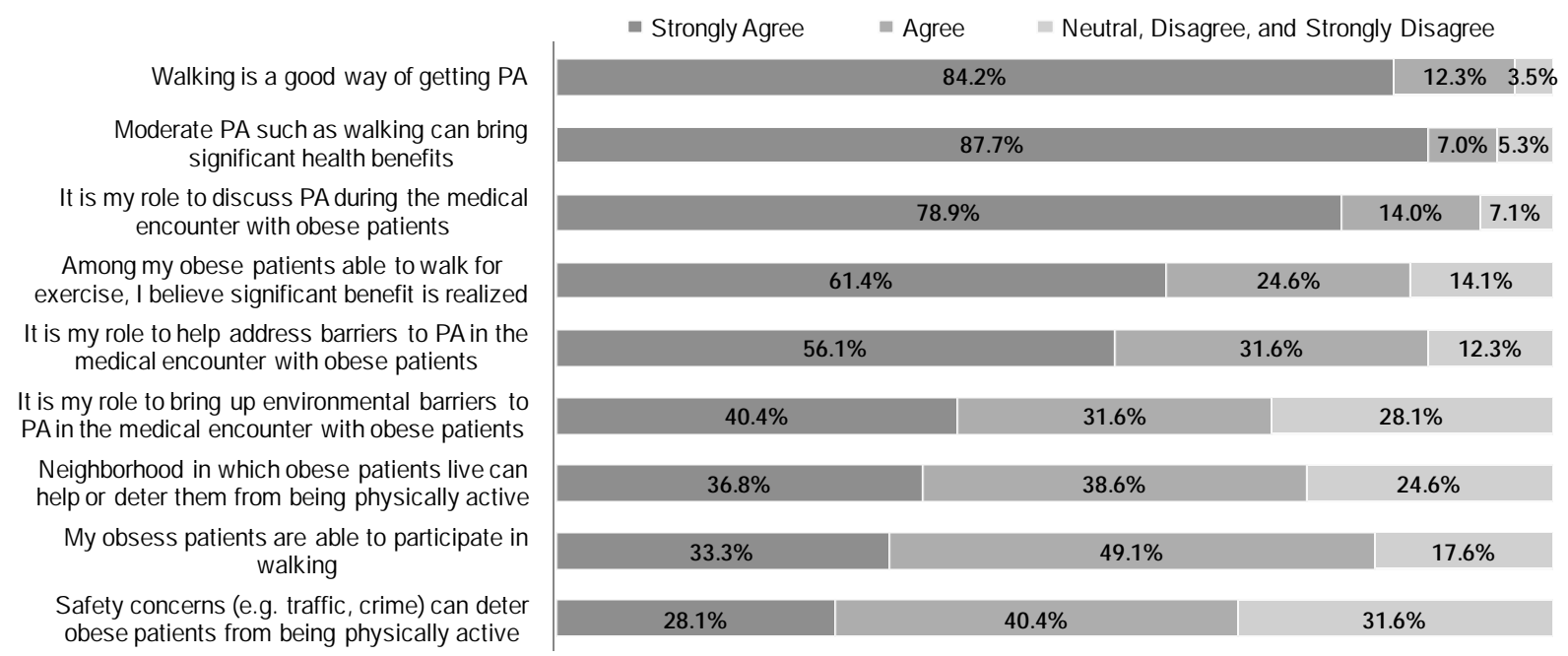

Figure 1. Physician perceptions about physical activity and the neighborhood environment.

\begin{tabular}{|c|c|c|c|c|}
\hline \multirow[b]{2}{*}{ Time in anyone encounter } & $=$ Very Important & = Somewhat Important & \multicolumn{2}{|c|}{$=$ Not at all Important } \\
\hline & \multicolumn{2}{|c|}{$56.1 \%$} & $35.1 \%$ & $8.8 \%$ \\
\hline Knowing specific strategies to recommend to & \multirow{2}{*}{$31.6 \%$} & \multirow{2}{*}{\multicolumn{2}{|c|}{$57.9 \%$}} & $10.5 \%$ \\
\hline patients to overcome environmental barriers & & & & 10.570 \\
\hline $\begin{array}{c}\text { Knowing where patients can be physically active } \\
\text { safely in their environments }\end{array}$ & $28.1 \%$ & $56.1 \%$ & \multicolumn{2}{|r|}{$15.8 \%$} \\
\hline Knowing what environmental factors influence PA & $14.3 \%$ & $67.9 \%$ & & $17.9 \%$ \\
\hline Concern that environment can't be changed & $7.1 \%$ & $64.3 \%$ & \multicolumn{2}{|c|}{$\mathbf{2 8 . 6 \%}$} \\
\hline Concern that patient won't be interested in topic & $17.9 \%$ & $46.4 \%$ & \multicolumn{2}{|c|}{$35.7 \%$} \\
\hline
\end{tabular}

Figure 2. Enabling factors for physician discussion of environmental issues with their patients.

The physicians generally had a positive interaction with their obese patients about physical activity and walking during the last few encounters as reported elsewhere [16]. Among the top activities most often discussed included asking whether their obese patients engaged in any physical activity (89.5\%), whether they advised their patients about meeting the physical activity recommendation of at least 30 minutes of physical activity on at least five days a week (75.4\%), as well as whether they walked for recreation, exercise, to get to and from places $(71.4 \%)$. Issues sometimes discussed included environmental barriers to physical activity and walking. The majority reported often or at least sometimes asking about environmental barriers to being more physically active $(82.5 \%)$ or walking more $(80.7 \%)$. The majority also reported that their obese patients often or sometimes brought up the environment as a barrier to being more physically active (91.2\%) or walking more (89.3\%), while $84.2 \%$ reported often or sometimes giving specific examples to overcome these barriers.

\subsection{Physician Recommendation of Physical Activity}

Walking was the most common activity recommended by the physicians for their obese patients; all 57 respondents recommended walking. Other activities frequently recommended included bicycling (82.5\%), swimming (82.5\%), and strength training (57.9\%). Activities less often recommended included gardening (28.1\%), dancing (15.8\%), water aerobics, yoga, and walking in the pool.

\subsection{Physician Perception on Physical Activity and the Environment}

Physicians identified a number of environmental barriers to being physically active especially important for their obese patients. The most prevalent environmental barriers mentioned in this open-ended question were weather (29.1\%) and safety (by 29.1\%). Lack of sidewalks, lack of trails/tracks, and inadequate walking surfaces were also reported by $14.5 \%, 14.5 \%$, and $12.7 \%$ of the physicians, respectively. Physicians reported the mall (45.6\%), sidewalks or neighborhoods (36.8\%), and parks (33.3\%) as good walking environments for their obese patients.

\section{DISCUSSION}

In this study, we explored the role of family physicians 
and their perceptions about environmental influences in promoting physical activity and walking in obese patients. In the population we surveyed, the majority of family physicians recognized the benefit of physical activity for health, even among their obese patients. In line with clinical guidelines [17], discussions about physical activity are now becoming integral routine part of ambulatory clinical practice.

Effective behavioral counseling in primary care settings calls for an awareness of the five A's, Assess, Advise, Agree, Assist, and Arrange [18]. This involves asking patients about their engagement in certain behaviors, advising them about desired goals, seeing if they agree, assisting them in identifying and overcoming barriers through evidence-based behavior change strategies, and arranging. While we found strong endorsement for general physical activity goal-setting and treatment plans, there was less agreement on the importance of discussing general barriers to physical activity, especially in regards to addressing environmental barriers. There was less attention to the use of written prescriptions or contracts which have been shown to be an effective strategy for promoting physical activity [19].

Modeling is another important behavior change strategy and while more physically active than the general adult population or those in Texas just slightly over half of the surveyed family physicians (56.3\%) were meeting the Surgeon General's recommended physical activity guidelines themselves. We are inclined to believe that physicians who are more physically active themselves might be more likely to prescribe exercise, indicating the importance of physicians meeting the recommended guidelines for their own health as well as being a role model for their patients.

In preparing for this research, we found sparse literature on the discussions about the physical environment in medical encounters. This research provides an initial glimpse at what physicians know about the physical environment as a facilitator or a barrier to physical activity, and how this knowledge is translated into clinical practice. We have documented reasons why this knowledge is not being translated into practice, with time being the major factor mentioned, as similarly reported in other studies examining lifestyle discussions in the medical encounter [9].

As with any exploratory study, there are a few limitations to be addressed. Among these are selection and recall bias. Although all family physicians were approached voluntarily to participate, not all of them did. It is likely that those who participated may have been more physically active than those who did not respond but we could not determine this since we did not have data on nonrespondents' physical activity. To limit recall bias associated with self-reports the survey was designed to limit responses to the last few patient-physician encounters. Moreover, while we were careful in the construction of the survey to emphasize that there were no "right or wrong” responses, self-reported data are always subject to the potential of socially desirable responses, and can not be verified without more costly record abstractions or actual clinical observations. In the survey, the word "sometimes" used in the "often-sometimes-never" 3-item scale may have appeared vague and somewhat subjective to the respondents. Providing more quantifiable, less subjective options could have removed the perceived vagueness.

The small sample size resulted in our inability to relate physician responses to physician characteristics (e.g., age, gender, and race/ethnicity) or to examine the interrelationships among study variables. The limitations of this study clearly point to the need to conduct future larger research to explore current physician practices and to identify best practices for assisting physicians in introducing environmental factors in discussions of lifestyle risk factors. Finally, this study involved only one health plan, which may limit its generalization to other settings outside our large integrated healthcare organization. However, working with CenTexNet enabled us to obtain responses from clinicians working in several different clinical settings throughout the system. Moreover, the involvement of the Family Medicine Department Leadership on the front end of the study combined with value put on research by CenTexNet members, resulted in an excellent response rate of nearly $60 \%$ which exceeds the typically low rates of other physician surveys [20].

In summary, we believe that physicians have an important role in helping their obese patients overcome barriers to physical activity. It is clear that most physicians have some knowledge about the environment and physical activity, but fewer do not routinely interact with their obese patients about the environmental concerns. Given our study findings documenting several enabling factors affecting physician discussions of environmental issues, we see a need for targeted information and provision of useful tools to assist physicians to help their obese patients. Many physicians believe that strategies to overcome environmental barriers are important and many would like to have knowledge about safe places near patients' home in order to refer them to engage in physical activity. Toward this end, study investigators have created a web-based resource guideline for family physicians that could be downloaded and given to patients. This includes a motivational cover page confirming the importance of physical activity for health, combined with a resource guide that points patients to places in their community where they could engage in various types of physical activity in safely and conveniently. 


\section{ACKNOWLEDGEMENTS}

This work was supported by a Scott \& White Institutional Research Fund \#070940 awarded to the lead author (SF). Portions of the data were presented at the 2009 Agency for Healthcare Research \& Quality National Practice-Based Research Network (PBRN) Research Conference, Bethesda, MD, June 24-26, 2009.

\section{REFERENCES}

[1] US Department of Health and Human Services (2008) Physical activity guidelines for Americans, 2008.

[2] Flegal, K.M., Carroll, M.D., Ogden, C.L. and Curtin, L.R. (2010) Prevalence and trends in obesity among US adults, 1999-2008. Journal of the American Medical Association, 303, 235-241. doi:10.1001/jama.2009.2014

[3] CDC (2009) Prevalence and trends data. Report No. 2011.

[4] US Department of Health and Human Services (2010) The surgeon general's vision for a healthy and fit nation. Public Health Reports, 125, 514-515.

[5] Rejeski, W.J., Brubaker, P.H., Goff, Jr., D.C., Bearon, L.B., McClelland, J.W., Perri, M.G. and Ambrosius, W.T. (2011) Translating weight loss and physical activity programs into the community to preserve mobility in older, obese adults in poor cardiovascular health. Archives of Internal Medicine, 171, 880-886.

doi:10.1001/archinternmed.2010.522

[6] Eyler, A.A., Bronson, R.C., Bacak, S.J. and Houseman, R.A. (2003) The epidemiology of walking for physical activity in the United States. Medicine \& Science in Sports \& Exercise, 35, 1529-1536. doi:10.1249/01.MSS.0000084622.39122.0C

[7] Anis, N.A., Lee, R.E., Ellerbeck, E.F., Nazir, N., Greiner, K.A. and Ahluwalia, J.S. (2004) Direct observation of physician counseling on dietary habits and exercise: Patient, physician, and office correlates. Preventive Medicine, 38, 198-202. doi:10.1016/j.ypmed.2003.09.046

[8] Ma, J., Urizar, Jr., G.G., Alehegn, T. and Stafford, R.S. (2004) Diet and physical activity counseling during ambulatory care visits in the United States. Preventive Medicine, 39, 815-822. doi:10.1016/j.ypmed.2004.03.006

[9] Ory, M.G., Peck, B.M., Browning, C. and Forjuoh, S.N. (2007) Lifestyle discussions during doctor-older patient interactions: The role of time in the medical encounter. Medscape General Medicine, 9, 48.

[10] Santariano, W., Ory, M. and Lee, C. (2012) Planned and built environments: Interactions with aging. Public Health in an Aging Society, The Johns Hopkins Press, Baltimore, In Press.

[11] Lee, C. (2007) Environment and active living: The roles of health risk and economic factors. American Journal of Health Promotion, 21, 293-304.

doi:10.4278/0890-1171-21.4s.293

[12] Lee, I.M. and Buchner, D.M. (2008) The importance of walking to public health. Medicine \& Science in Sports \& Exercise, 40, S512-518. doi:10.1249/MSS.0b013e31817c65d0

[13] Forsyth, A., Brownson, R.C. and Day, K. (2009) The built environment, walking and physical activity: Is the environment more important to some people than others? Transportation Research Part D: Transport and Environment, 14, 42-49. doi:10.1016/j.trd.2008.10.003

[14] Popkin, B.M., Duffey, K. and Gordon-Larsen, P. (2005) Environmental influences on food choices, physical activity and energy balance. Physiology \& Behavior, 86, 603-613. doi:10.1016/j.physbeh.2005.08.051

[15] Phelan, S., Nallari, M., Darroch, F.E. and Wing, R.R. (2009) What do physician recommend to their overweight and obese patients? Journal of the American Board of Family Medicine, 22, 115-122. doi:10.3122/jabfm.2009.02.080081

[16] Forjuoh, S.N., Lee, C., Wang, S., Hong, Y. and Ory, M.G. (2011) Patient-physician discussion of physical activity and environmental barriers. Preventive Medicine, 53, 209210. doi:10.1016/j.ypmed.2011.07.010

[17] (1998) Clinical guidelines on the identification, evaluation, and treatment of overweight and obesity in adultsThe evidence report. National Institutes of Health. Obesity Research, 6, 51S-209S.

[18] Glasgow, R.E., Emont, S. and Miller, D.C. (2006) Assessing delivery of the five "As" for patient-centered counseling. Health Promotion International, 21, 245-55. doi:10.1093/heapro/dal017

[19] Cress, M.E., Buchner, D.M., Prochaska, T., et al. (2005) Best practices for physical activity programs and behavior counseling in older adult populations. Journal of Aging and Physical Activity, 13, 61-74.

[20] VanGeest, J.B., Johnson, T.P. and Welch, V.L. (2007) Methodologies for improving response rates in surveys of physicians: A systematic review. Evaluation \& the Health Professions, 30, 303-321. doi:10.1177/0163278707307899 